
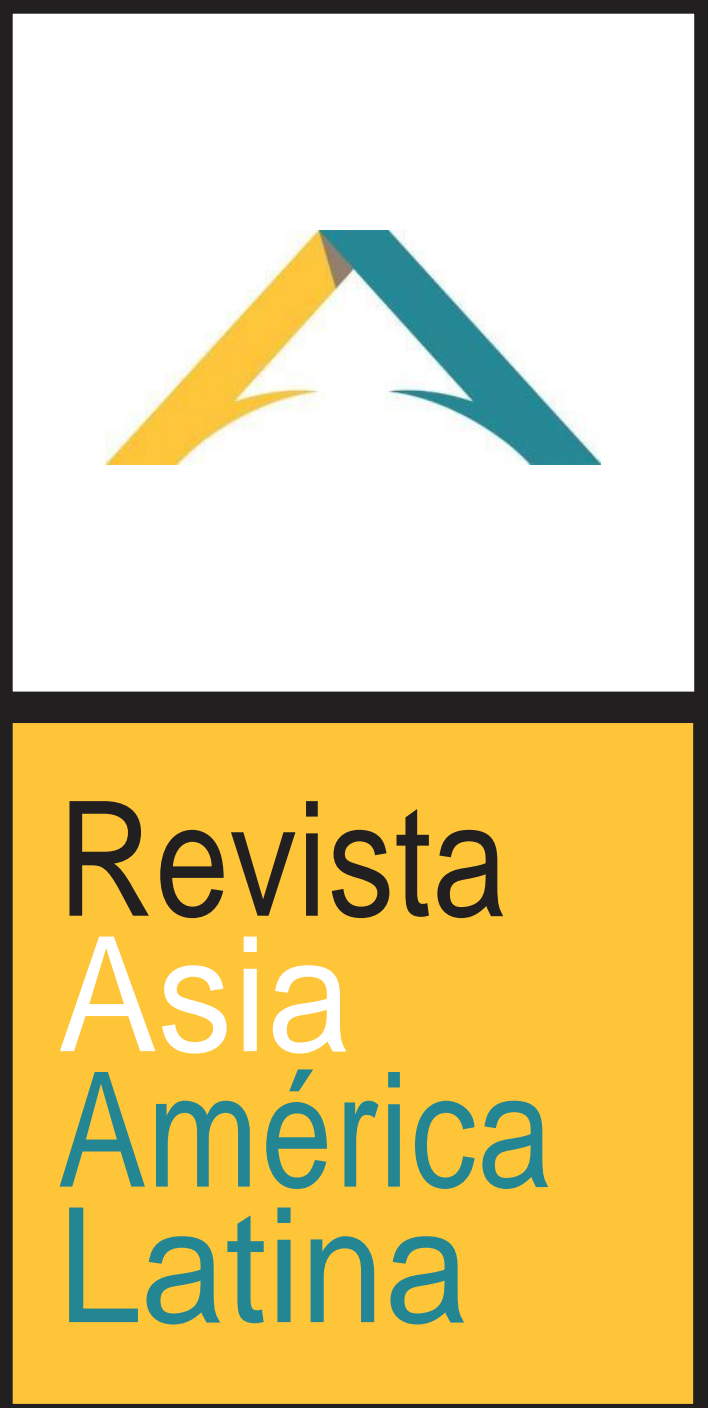

ISSN 2524-9347

Grupo de Estudios sobre Asia y América Latina Instituto de Estudios de América Latina y el Caribe Universidad de Buenos Aires

EU

DE BA 


\title{
INTERVENIR O NO INTERVENIR: EL DESAFÍO DE CREAR UN SISTEMA REGIONAL DE DERECHOS HUMANOS EN LA ASEAN
}

\author{
Alejandro Lamarque \\ Universidad de Buenos Aires \\ alejandro.lamarque@gmail.com
}

\section{Introducción}

El himno oficial de la Asociación de Naciones del Sudeste Asiático (ASEAN por sus siglas en inglés) sintetiza en pocas estrofas el espíritu de un proyecto de integración regional de más de cinco décadas. Unidad, prosperidad y paz son las metas compartidas por un conjunto de naciones ante el desafío de posicionarse en un sistema de relaciones internacionales poblado de viejas y nuevas potencias. Es así como la ASEAN devino el medio a través del cual la región adquiere identidad propia, vertebrando su proyección e inserción en el escenario global.

Con el tiempo, el interés internacional por el bloque ASEAN ha destacado su capacidad de sentar bases firmes para la cooperación entre los países de una región marcada por su diversidad política, geográfica, religiosa, étnica y económica. Ante todo, los admirables desempeños económicos de la región han predominado en las discusiones sobre esta iniciativa regional. Sin embargo, tal como indica la quinta estrofa de su himno oficial - For peace, our goal from the very start"-, los primeros avances y éxitos de la ASEAN no fueron en cuestiones económicas sino en cuestiones securitarias.

Este ensayo propone tomar en consideraciones los alcances y limitaciones de este nuevo sistema regional de derechos humanos. Para ello, la primera parte detalla el desarrollo de la ASEAN como respuesta a imperativos de seguridad en una región inestable. La atención se centra en contextualizar la importancia del principio de no intervención, mostrando su incidencia en cada desarrollo o iniciativa de la asociación. Luego se procede a describir el desarrollo del incipiente sistema de derechos humanos en un período de profundo cambio institucional. Por último, la tercera parte puntualiza las limitaciones del sistema regional de derechos humanos como cristalización de dos perspectivas en disputa. 
Intervenir o no intervenir: el desafío de crear un sistema regional de derechos

humanos en la ASEAN

ALEJANDRO LAMARQUE

\section{Orígenes de ASEAN: el principio de no intervención como fundamento de la seguridad regional}

Tras su creación en 1967, las prioridades de la ASEAN no podían ser otras que no fueran la seguridad regional. ${ }^{1}$ El contexto de inestabilidad política nacional y regional en las primeras décadas de posguerra fue uno de los problemas compartidos por cada uno de los Estados del Sudeste Asiático (SEA), y fue anterior al comienzo del proceso de integración. Así las jóvenes naciones de la región emprendieron la formación de una organización regional capaz de aliviar tensiones y facilitar la discusión, contención y resolución pacífica de los conflictos entre Estados.

A lo largo de cinco décadas de existencia, la ASEAN se constituyó en una instancia articuladora de la seguridad regional en el SEA. Esto no es una cuestión menor, dado el pasado de desconfianza y enfrentamientos entre los países de la región. En igual medida, la incertidumbre respecto a la paz y seguridad sigue siendo hasta hoy de suma importancia, no solo para los Estados, sino también para sus poblaciones que han vivido procesos de violencia extrema.

Como indica Bellamy (2014), durante la Guerra Fría la región fue un epicentro de los dos grandes conflictos: por una parte, la lucha entre ideologías comunistas y anticomunistas y por otra, entre la descolonización y la construcción de los Estados nacionales. En el contexto de estos conflictos, la violencia extrema contra civiles llegó a ser considerada una herramienta para asegurar los objetivos planteados a un costo "razonable".

La región vivió picos de violencia en las décadas de los años 60 y 70 con el asesinato de opositores -presuntos comunistas- en Indonesia y Filipinas, la intensificación de la guerra en Indochina, la invasión Indonesia a Timor Leste, las campañas contra milicias étnicas en Myanmar, el régimen de Kampuchea Democrática en Camboya y una multiplicidad de conflictos étnicoreligiosos e independentistas en regiones como Aceh, Papúa Occidental, Sulawesi y Molucas, Mindanao, sur de Tailandia, Rakhine, Sabah y Sarawak (Bellamy, 2014; Cribb, 2013; Urgell, 2007).

Con el tiempo y bajo el influjo de las circunstancias, la ASEAN se reformó para adaptarse a los nuevos desafíos que se abrían con los cambios geopolíticos y sus influencias en la región. Simultáneamente, a la puesta en marcha de una Comunidad ASEAN, la Asociación dio pasos firmes y rápidos en la creación de un sistema regional de derechos humanos. Esto fue tomado con sorpresa e incluso escepticismo por parte de la comunidad internacional.

1 Para profundizar en el tema ver Rubiolo, 2006; Tang, Wilhelmy von Wolff \& Fajardo Vallejo, 2007; Kelin, Som, Saludin, Shuib \& Ajis, 2011; Davies, 2013; Bartesaghi, 2014 y Emmers, 2016. 
Intervenir o no intervenir: el desafío de crear un sistema regional de derechos

humanos en la ASEAN

ALEJANDRO LAMARQUE

Los instrumentos y dispositivos desarrollados hasta la fecha se han desarrollado no sin dificultades, ya que cristalizan las tensiones entre dos principios: por una parte, el de no intervención que estructura cada documento, práctica e institución de ASEAN. Por otra, la responsabilidad de proteger que se ha afianzado como norma internacional y genera expectativas sobre cómo responder ante el genocidio y los crímenes masivos (Bellamy, 2015). El resultado de ésta "síntesis", como la denomina Davies (2013), resulta en un sistema de derechos humanos diferente al de otras regiones; condicionado por las tensiones entre esos dos principios distintos.

La ASEAN fue el último en una serie de intentos infructuosos de crear instancias regionales de cooperación para los Estados del Sudeste Asiático. Entre fines de los 50s y 60s, se sucedieron distintas iniciativas que variaron en su composición y prioridades. Tal es el caso la Association of Southeast Asia (ASA), creada el 31 de julio de 1961 e integrada por Malasia, Filipinas y Tailandia. Esta asociación pensada para alcanzar paz y estabilidad regional, al igual que cooperación cultural y económica, llegó a su fin por las disputas territoriales de sus miembros en torno a la región de Sabah.

Posteriormente, ASA fue sucedida por MAPHILINDO sigla que hace referencia al acuerdo alcanzado por Malasia, Filipinas (Philippines) e Indonesia en julio de 1963. Esta experiencia regional también llegaría a su fin de la mano de la Konfrontasi entre Malasia e Indonesia respecto a Borneo. No fue hasta la caída del gobierno de Sukarno en Indonesia, en 1967, que el peligro de la confrontación quedó aplacado y se dio una convergencia sobre los beneficios de una organización regional. Esto se materializó en la Declaración de Bangkok que creó la Asociación de Naciones del Sudeste Asiático (ASEAN) con la firma de Malasia, Singapur, Indonesia, Filipinas y Tailandia el 8 de agosto de $1967 .^{2}$

Al surgir en un contexto de grandes tensiones regionales, la ASEAN fue ante todo un foro para forjar confianza entre sus miembros y establecer normas de convivencia. Así, del modo en que los asuntos eran discutidos, las diferencias negociadas y las decisiones tomadas surgió la cultura diplomática del Sudeste Asiático conocida como "ASEAN Way". Como indican Chua y Lim (2018), el rasgo primario de esta cultura es la informalidad de las interacciones entre países, facilitando flexibilidad en las formas de tramitar problemas y tensiones. Esta impronta informal caracterizó el desarrollo institucional de ASEAN durante varias décadas, hasta que la ampliación de sus integrantes y de sus objetivos exigieron una institucionalidad formal firme.

Las características predominantes del estilo ASEAN son la centralidad de las decisiones por consenso y el principio de no intervención en los asuntos de otros Estados tanto de potencias extrarregionales como de países de la

2 Para profundizar ver Tang, Wilhelmy von Wolff \& Fajardo Vallejo, 2007 y Kelin, Som, Saludin, Shuib \& Ajis, 2011. 
Intervenir o no intervenir: el desafío de crear un sistema regional de derechos

humanos en la ASEAN

ALEJANDRO LAMARQUE

región. Dichos asuntos permanecen rigurosamente excluidos en la medida que no tengan incidencia en la estabilidad regional. Este principio se consolidó como el fundamento de la ASEAN con la firma, en 1971, de la Declaración de Kuala Lumpur que establece la Zona de Paz, Libertad y Neutralidad (ZOPFAN). Esta Declaración muestra la voluntad de las cinco naciones de ASEAN de asegurar la región frente a intervenciones de potencias extranjeras a medida que la Doctrina Guam del Presidente Nixon anunciaba la retirada de los Estados Unidos del SEA y la influencia sino- soviética se afirmaba en Indochina (Rubiolo, 2006).

Como sugiere Rubiolo, desde una perspectiva constructivista, el principio de no intervención es un aspecto de la estructura de conocimiento compartido sobre el que se establecen relaciones de cooperación y se define el rol de cada actor. Así, es "el consenso en torno a la primacía de este principio el que permite un nuevo avance hacia el abandono de la conflictividad, y la búsqueda de soluciones de manera conjunta” (Rubiolo, 2006, pp. 23).

Posteriormente, este principio sería reforzado con la firma del Tratado de Amistad y Cooperación (TAC) en la Cumbre de Bali de 1976. Este es un pacto de no agresión entre sus miembros que institucionaliza pautas de conducta como el respeto por la independencia, la soberanía y la integridad territorial, la no intervención en los asuntos internos y la solución pacífica de las controversias (Tang, Wilhelmy von Wolff \& Fajardo Vallejo, 2007). Retomando a Chua y Lim (2018), el TAC hasta el día de hoy es un documento esencial en las relaciones de ASEAN con socios extrarregionales y por ello, las naciones que busquen entablar vínculos de cooperación con ASEAN deben respetar los principios delineados por ese tratado.

La firma del TAC coincide con la intensificación del conflicto en Indochina, que con el tiempo se convertiría en la prueba de fuego de la ASEAN. La frágil estabilidad de la región se vio sacudida en el año 1975 cuando Vietnam avanzó sobre el resto del país tras la retirada de EE.UU. Ese mismo año, el movimiento comunista camboyano liderado por Pol Pot derrotó a las fuerzas del mariscal Lon Nol e inició un proyecto de transformación radical que tendría devastadoras consecuencias para su población.

En el mismo año en que se firmó el TAC, los países de ASEAN firmaron la Declaración de la "Concordia ASEAN". Ambos documentos son un llamado a la resolución pacífica de controversias bajo la sombra amenazante de una expansión del conflicto en Indochina (Rubiolo, 2006). El momento crítico llegó con la invasión de Vietnam a Camboya en diciembre de 1978 tras dos años de enfrentamientos fronterizos. Tal como lo indica la declaración conjunta de la reunión especial de representantes emitida el 12 de enero de 1979, esta invasión violó normas básicas del TAC, como el principio de no intervención, la renuncia al uso de la fuerza y la resolución pacífica de conflictos. Simultáneamente, China invadió Vietnam para salir derrotada poco 
Intervenir o no intervenir: el desafío de crear un sistema regional de derechos

humanos en la ASEAN

ALEJANDRO LAMARQUE

tiempo después sin siquiera comprometer la presencia vietnamita en Camboya. Se inició de este modo un conflicto que marcaría la dinámica regional durante una década y posicionaría a la ASEAN como articulador de la política del SEA ante a los ojos de la comunidad internacional (Chua \& Lim, 2018).

En junio de 1980, Vietnam atacó el pueblo tailandés de Ban Non Mak Moon cerca de la frontera camboyana hasta que la Fuerza Aérea y el Ejército tailandés expulsaron a las fuerzas vietnamitas. Así se concretaban los temores de la ASEAN de un spillover del conflicto en toda la región. Ante la concreción de la amenaza, ASEAN desarrolló una respuesta concertada para la crisis camboyana que como indican Chua y Lim (2018) consistió en tres pilares: primero, el apoyo a las fuerzas nacionalistas de Camboya -como las lideradas por el Príncipe Sihanouk- en contra de la ocupación vietnamita. En segundo lugar, aislar a Vietnam y ejercer presiones diplomáticas, económicas y políticas para entablar negociaciones y alcanzar una solución política que incluyera la retirada total de las fuerzas vietnamitas y la realización de elección libres bajo supervisión internacional. Por último, la búsqueda de una solución política que devolviera a Camboya su soberanía e independencia pero que también atendiera las preocupaciones legítimas de sus vecinos, incluyendo a Vietnam, sobre cuestiones de seguridad.

Más allá de sus divergencias, los cinco países de la ASEAN actuaron concertadamente, presentando con éxito resoluciones de la Asamblea General de Naciones Unidas e impidiendo el reconocimiento del gobierno provietnamita establecido en Camboya. Posteriormente, ASEAN auspició las reuniones informales de Yakarta (Jakarta Informal Meeting I y II) de 1989 que contribuyeron al desarrollo de la Conferencia de París sobre Camboya.

Ese mismo año, ante los efectos de las sanciones económicas y la asistencia menguante de la URSS, Vietnam se vio forzada a retirar sus fuerzas del país vecino. El conflicto culminó con la firma de los Acuerdos de París en 1991 y la ASEAN ganó reconocimiento internacional por sus contribuciones al poner la cuestión camboyana en la agenda internacional y buscando una salida política (Rubiolo, 2006).

Tras la firma de los Acuerdos de Paz, Naciones Unidas asumió la responsabilidad de asegurar una transición pacífica. La Autoridad Provisional de las Naciones Unidas en Camboya asumió las responsabilidades de gobierno y la organización de las primeras elecciones nacionales. También en este proceso, las naciones de ASEAN contribuyeron con personal militar y policial al igual que asesores diplomáticos para asistir al Consejo Supremo Nacional de Camboya liderado por su presidente, el Príncipe Sihanouk (Merican, 2007).

Retomando a Rubiolo (2006), la ASEAN encontró en la gestión del conflicto de Camboya la necesidad de alcanzar unidad, demostrando que el estilo $A S E A N$ de intervenir en conflictos podía brindar respuestas efectivas y decisivas a pesar de las diferencias internas entre cada uno de sus países. Hechos 
Intervenir o no intervenir: el desafío de crear un sistema regional de derechos

humanos en la ASEAN

ALEJANDRO LAMARQUE

concretos como la amenaza vietnamita para Tailandia movilizaron esa posición común por parte de la ASEAN a la vez que los Estados miembros demostraron voluntad de actuar más allá de sus intereses particulares en defensa de la estabilidad regional.

Las mismas funciones de mediación tuvieron lugar en la crisis política de 1997 iniciada por el desplazamiento del Primer Ministro Norodom Ranariddh de la coalición de gobierno por el entonces Co-Primer Ministro Hun Sen. Esta crisis demoró el ingreso de Camboya a la ASEAN, llevando a la creación de la llamada Troika ASEAN (instancia ad-hoc compuesta por tres presidentes del Comité Permanente) para preservar los logros de la UNTAC.

Asimismo, en el año 2011 ASEAN intervino en la disputa fronteriza entre Camboya y Tailandia por la posesión del templo Preah Vihear que incluyó la movilización de tropas y el desplazamiento de miles de habitantes que escaparon de los enfrentamientos. El conflicto comenzó en 2008 pero la ASEAN se abstuvo de intervenir a instancias de los países en disputa que quisieron evitar la internacionalización del conflicto. Sin embargo, a medida que los mecanismos bilaterales fracasaban y el conflicto se agudizaba, el Consejo de Seguridad de Naciones Unidas decidió ceder la responsabilidad de resolver el conflicto a la ASEAN (UN Security Council, 2011). Tras mantener reuniones con las partes, ASEAN decidió enviar observadores de la asociación para supervisar las negociaciones. Si bien la reticencia de Tailandia frustró esta iniciativa, la delegación de responsabilidad de Naciones Unidas a la ASEAN no tenía precedentes en la historia de la organización (Amador \& Teodoro, 2016).

Los instrumentos firmados por los países miembros al igual que su intervención conjunta en momentos de inestabilidad regional evidencian que la función primaria de ASEAN como organización regional es la preservación de la seguridad en el SEA. Como indican Amador y Teodoro (2016), las organizaciones regionales tienen un rol en cuestiones de seguridad internacional porque las regiones son el punto de encuentro entre seguridad nacional y seguridad global.

Los conflictos al interior de los Estados no pueden ser disociados de su ambiente externo y la seguridad de un Estado en particular es inseparable de la seguridad de sus vecinos. La formación de arreglos regionales mediante organizaciones como ASEAN confirma este consenso.

\section{Institucionalización de la ASEAN: hacia una comunidad y el desarrollo de un sistema de derechos humanos}

Como se indicó en la introducción, este ensayo se centra en cuestiones de seguridad y derechos humanos en el marco de la ASEAN por sobre las cuestiones económicas que, a menudo, han sido las más tratadas. Teniendo en 
Intervenir o no intervenir: el desafío de crear un sistema regional de derechos

humanos en la ASEAN

ALEJANDRO LAMARQUE

cuenta esto, bastará mencionar que la paz iniciada luego de la crisis camboyana permitió retomar las metas económicas postergadas durante varias décadas. Así en 1992 los países de la ASEAN se comprometieron a establecer y participar del Área Exclusiva de Libre Comercio (AFTA) y el Acuerdo Marco para el fortalecimiento de la Cooperación Económica en ASEAN. Estos pasos son precursores de los objetivos económicos definidos en la Segunda Declaración de la Concordia que significó la creación de una Comunidad Económica de ASEAN. También se crearon instancias de cooperación con las grandes economías de la región como el llamado ASEAN Más Tres (APT) (Rubiolo, 2006).

La solución del conflicto camboyano abrió una etapa de expansión de la ASEAN hasta incluir a todos los Estados del SEA. Como indican Chua y Lim (2018), la ASEAN fue pensada desde su concepción como una instancia regional inclusiva que incorporara a todos los países del SEA. Hasta no conseguirlo, la ASEAN sería considerada una organización subregional. La ampliación habilitaría que todos los miembros compartieran instancias de diálogo y códigos de conducta comunes para alcanzar sus metas de prosperidad económica y seguridad política. Por eso, a la previa incorporación de Brunéi Darussalam en 1984 siguieron Vietnam (1995), Laos, Myanmar (1997) y Camboya (1999). Timor Leste participó como invitado en varias reuniones especiales de ASEAN, solicitando formalmente convertirse en el onceavo miembro en 2013, aunque todavía no lo ha logrado.

En este período ASEAN no solo expandió su número de miembros sino también su número de socios extrarregionales. La creciente presencia China en la región impulsó la formación de instancias de cooperación multilateral para minimizar posibles enfrentamientos como las tensiones surgidas respecto a la propiedad de las islas del Mar de China Meridional y la península coreana, entre otros.

En función de lo antedicho, en 1994 se creó el Foro Regional de ASEAN (ARF) cuyo programa contempla tres fases: construcción de confianza, diplomacia preventiva y solución de conflictos. ${ }^{3}$ El foro también analiza problemas de seguridad no convencionales como el terrorismo y el crimen internacional. Además, aspirando a conseguir interdependencia en materia de seguridad, el foro promovió el Tratado de Amistad y Cooperación y el código de conducta estándar para las relaciones interestatales (Tang, Wilhelmy von Wolff \& Fajardo Vallejo, 2007).

${ }^{3}$ En la actualidad, los miembros extrarregionales que participan del foro son Australia, Bangladesh, Canadá, China, República Popular Democrática de Corea, Unión Europea, India, Japón, Mongolia, Nueva Zelanda, Pakistán, Papúa Nueva Guinea, República de Corea, Rusia, Sri Lanka y Estados Unidos. 
Intervenir o no intervenir: el desafío de crear un sistema regional de derechos

humanos en la ASEAN

ALEJANDRO LAMARQUE

Hasta entonces, el desarrollo de las funciones de la ASEAN se dio sobre el entramado informal y flexible de relaciones que caracteriza al estilo $A S E A N$. Sin embargo, los eventos que marcaron los últimos años del siglo sacudieron los consensos básicos de esta cultura diplomática e incentivaron procesos de reforma.

Hacia fines de 1997, la crisis asiática evidenció la persistente vulnerabilidad de la región a influencias externas. La capacidad de ASEAN de lidiar conjuntamente con problemas de tal envergadura como los resultantes de la globalización fue puesta en tela de juicio. Asimismo, la asociación fue criticada por no atender eficazmente cuestiones relativas a los derechos humanos y problemas transnacionales como desastres naturales.

En ese ambiente de cuestionamientos, surgieron debates sobre los alcances del principio de no intervención y la institucionalización de la Asociación. Se alzaron voces que los consideraron inadecuados para fundamentar las bases de un orden regional Indo-Pacífico (Acharya, 2009; Davies, 2013, 2014).

El consenso sobre la necesidad de reformas se empezó a manifestar en diciembre de 1997, cuando los representantes de los países miembros firmaron el documento de la Visión de ASEAN 2020. Esta visión plasma un renovado esfuerzo por fortalecer los lazos de integración de la región y por construir una identidad compartida sobre la cual fundar una comunidad. En 2003, se firmó la Declaración de Concordia en Bali, donde se detalla formalmente el establecimiento de la Comunidad ASEAN. Dicha comunidad descansa sobre tres pilares: la cooperación política y de seguridad, la cooperación económica, y la cooperación socio cultura.

Por último, la transición de un modo informal de cooperación hacia un marco institucionalizado se dio mediante una constitución que diera entidad legal a la Asociación para entablar acuerdos vinculantes con sus socios y otras organizaciones internacionales. Con la entrada en vigencia de la Carta en 2008 comienza una nueva etapa de la ASEAN. Se dejan atrás los vínculos flexibles e informales en favor de relaciones institucionalizadas respaldadas por instrumentos codificados vinculantes.

Es durante este clima de discusión de objetivos y procedimientos institucionales, que la ASEAN comienza a desarrollar un sistema regional de derechos humanos como los de Europa, África y las Américas. Es claro, que los derechos humanos no han sido una preocupación prioritaria de ASEAN, sin embargo, en las últimas dos décadas, la Asociación ha desarrollado instrumentos y dispositivos, culminando en una Declaración de Derechos Humanos, sobre la que se fundamenta un incipiente sistema de derechos humanos en el SEA.

Los antecedentes de la Declaración se remontan a la Conferencia Mundial de Derechos Humanos de 1993 realizada en Viena. Allí la ASEAN 
Intervenir o no intervenir: el desafío de crear un sistema regional de derechos

humanos en la ASEAN

ALEJANDRO LAMARQUE

produjo un comunicado conjunto en el que los países miembros se comprometían a considerar el establecimiento de un mecanismo regional de derechos humanos. Posteriormente, la crisis financiera de 1997 y sus graves consecuencias sociales reposiciona a los derechos humanos en las discusiones respecto a reformas institucionales dentro de la asociación.

Como indica Davies (2013), los miembros de ASEAN se dividieron en dos extremos en un espectro progresista-tradicionalista. El extremo progresista atribuyó el fracaso de lidiar con las causas y consecuencias de la crisis a la reticencia de comentar los asuntos internos de los demás Estados. Por otra parte, si bien los tradicionalistas no rechazaron de cuajo todo cambio, mantuvieron una firme decisión de alterar los compromisos con la soberanía estatal y la seguridad vigentes hasta la crisis.

Esta división explica en parte la ausencia de referencias a los derechos humanos en las declaraciones y tratados de la organización. La Visión 2020, el Grupo de Personas Eminentes (EPG) y los Acuerdos de Bali hacen referencias genéricas al bienestar de las sociedades del SEA. Es recién con la continuación de los Acuerdos de Bali, el Programa de Acción de Vientiane, que los derechos humanos comienzan a tener una presencia constante en las discusiones sobre reforma institucional.

En este programa los Estados de ASEAN acuerdan compartir información sobre derechos humanos, poner en marcha programas de trabajo al respecto, articular los mecanismos ya existentes sobre la materia y formar comisiones en defensa de los derechos de las mujeres, los niños y los migrantes (Davies, 2013 y 2014).

Algo determinante en todo este proceso fue enmarcar todas estas discusiones e iniciativas dentro del área de trabajo de la Comunidad de Política y Seguridad. Como indica Davies: "Dentro del pilar de Seguridad los derechos humanos fueron "vendidos" con éxito como algo no radicalmente nuevo, sino como una continuación lógica del compromiso tradicional de la ASEAN con asuntos de seguridad estatal" (Davies, 2013: 53). ${ }^{4}$ Así se llegó a la concreción de los elementos centrales del sistema de derechos humanos: la Declaración de Derechos Humanos ASEAN (AHRD) en 2012 y la Comisión Intergubernamental de Derechos Humanos de la ASEAN (AICHR) en 2009.

\section{Equilibrio entre intervención y no intervención: las limitaciones de los elementos del sistema de derechos humanos de ASEAN}

La "síntesis" descrita por Davies, entre perspectivas progresistas y tradicionalistas, en la creación del sistema regional de derechos humanos

\footnotetext{
${ }^{4} \mathrm{La}$ traducción del inglés al español es del autor.
} 
Intervenir o no intervenir: el desafío de crear un sistema regional de derechos

humanos en la ASEAN

ALEJANDRO LAMARQUE

resultó en la conformación de instrumentos y dispositivos con capacidades limitadas de intervenir en defensa de los derechos humanos de los habitantes del SEA. Como indica Bui (2016) en su análisis de la AHRD y la AICHR, esas restricciones son tanto de índole sustantiva como procedimental.

La principal limitación sustantiva radica en elementos que ponen en cuestión el principio de universalidad de los derechos humanos. A pesar que varios Estados de la región adoptaron tratados y declaraciones de derechos humanos fundados en el principio de universalidad, los gobiernos sostienen ideas particularistas y relativistas.

La AHRD queda condicionada en la medida que no se comprometa el contexto político, económico, religioso y cultural del Estado nacional. Esto en parte ha revivido el viejo debate sobre "valores asiáticos", utilizando argumentos culturalistas y particularistas para eximir a la región de estándares internacionales en distintos rubros (Bui, 2016; Davies, 2013 y 2014).

Por otra parte, los derechos individuales consagrados en estos instrumentos se encuentran balanceados por deberes sociales lo que supone límites claros a la posibilidad de exigir su realización y al deber de protegerlos. Esto quiebra un tema subyacente en los principios universales de derechos humanos ya que los individuos son poseedores de derechos y los Estados son los responsables de garantizarlos. En línea con los argumentos culturalistas, Bui (2016) identifica la contraposición de un presunto "comunitarismo" de las sociedades asiáticos por sobre un "individualismo" de las sociedades occidentales donde deberes no especificados hacia la comunidad condicionarían la defensa de derechos individuales.

Por último, la AHRD condiciona los derechos y libertades ante los imperativos de preservar el estado de derecho, la seguridad nacional y el orden público de los países en cuestión. De este modo, y como sugieren Bui (2016) y Davies (2013 y 2014), los derechos contenidos en la declaración de una entidad regional quedan supeditados a los mandatos y necesidades de las leyes domésticas.

Estas limitaciones serias al potencial del sistema regional de derechos humanos se ven agravadas por las limitaciones a los mecanismos diseñados para su protección. Bui (2016) describe el caso de la AICHR destacando, en primer lugar, su carácter de órgano de consulta intergubernamental lo que dificulta las posibilidades de ejercer accountability en materia de derechos humanos ya que su labor tendría que realizarse con los mismos Estados que debe controlar. Si bien no se puede decir que la Comisión carece de herramientas, estas están condicionadas por la falta de independencia respecto de los gobiernos de ASEAN.

En resumen, la "síntesis" descrita por Davies ha sumido al sistema regional de derechos humanos en un equilibrio entre la seguridad nacional y la seguridad de las personas donde la primera impera sobre la segunda. El 
Intervenir o no intervenir: el desafío de crear un sistema regional de derechos

humanos en la ASEAN

ALEJANDRO LAMARQUE

principio de no intervención continúa estructurando el desarrollo de la Asociación por sobre otras iniciativas de reforma.

\section{Conclusiones}

La historia de ASEAN es la historia de una región que intenta dejar atrás su pasado de inestabilidad política, conflictos interestatales y violencia extrema. Estos desafíos han vertebrado la formación de una empresa destinada a cambiar la lógica imperante en las relaciones internacionales de la región. En dicho camino, el principio de no intervención garantizó la unidad de los miembros en tiempos de crisis, sustituyendo los enfrentamientos por la resolución pacífica y consensuada de los conflictos.

Desde la solución del conflicto de Camboya no ha habido conflictos interestatales graves, confirmando la transformación de la región en un espacio de paz. Sin embargo, un distanciamiento del escenario regional permite ver los desafíos securitarios al interior de las fronteras, donde conflictos viejos y nuevos comprometen los derechos humanos más básicos de los pueblos del SEA. Así como la seguridad estatal no puede separarse de la seguridad regional, la seguridad de los pueblos al interior de las fronteras no debe ser ignorada. La dinámica de los acontecimientos traumáticos guio los impulsos de formación y reforma de la ASEAN.

Queda por ver si un sistema de derechos humanos incapaz de atender las demandas de los pueblos, no será obsoleto ante violaciones de derechos humanos. Violaciones que, de no ser tomadas en cuenta, puedan derivar en conflictos de alcance regional.

\section{Referencias bibliográficas:}

Acharya, A. (2009). Constructing a Security Community in Southeast Asia. ASEAN and the problem of regional order. Routledge, Londres.

AjIS, M.N.; Keling, M.F.; SAludin, M.H.; SHuib, S. y SOM, H. (2011). The Development of ASEAN from Historical Approach, Asian Social Science, Vol.7, No.7, pp. 169-189. Canadian Center of Science and Education.

BARTESAGHI, I. (2014). La ASEAN y el MERCOSUR: similitudes, diferencias y potencialidades, Revista Temas de Derecho Económico. Enfoque Nacional e Internacional, $N^{\circ} 2$, pp. 1-20. Tribunales Ediciones, Buenos Aires, Argentina.

Bellamy, A.J. (2014). The other Asian miracle? The decline of mass atrocities in East Asia, Global Change, Peace \& Security, Vol. 26, No.1, pp. 1-19. Recuperado 
Intervenir o no intervenir: el desafío de crear un sistema regional de derechos humanos en la ASEAN

ALEJANDRO LAMARQUE

https://www.tandfonline.com/doi/abs/10.1080/14781158.2014.86560 0

Bellamy, A.J. (2015). The responsibility to protect turns ten, Ethics \& International Affairs, Vol.29, No.2, pp. 161-185. Carnegie Council for Ethics in International Affairs. Recuperado de: https://www.cambridge.org/core/journals/ethics-and-internationalaffairs/article/responsibility-to-protect-turnsten/ACF97A60B5553FA72E5C4E015FBDB8E7

Bui, H. (2016). The ASEAN Human Rights System: A Critical Analysis, Asian Journal of Comparative Law, II, p. 111-140. National University of Singapore. Recuperado de: https://www.cambridge.org/core/journals/asian-journal-ofcomparative-law/article/asean- human-rights-system-a-criticalanalysis/095F2F8A32A544F1AEEB6A2D89328F1E

CHUA, D. y LIM, E. (2018). ASEAN 50. Regional Security Cooperation though selected documents. S. Rajaratnam School of International Studies. Recuperado de: https://www.worldscientific.com/worldscibooks/10.1142/1047 8

CribB, R. (2013). Political Genocide in Postcolonial Asia, en Bloxham, D. y Dirk Moses, A. (Eds.), The Oxford Handbook of Genocide Studies, pp. 445465. Oxford University Press.

DAvies, M. (2013). The ASEAN Synthesis: Human rights, Nonintervention, and the ASEAN Human Rights Declaration, Georgetown Journal of International Affairs, Vol. 14, No. 2, pp. 51-58. Recuperado de: https://www.georgetownjournalofinternationalaffairs.org/onlineedition/the-asean-synthesis- human-rights-non-intervention-andthe-asean-human-rights-declaration-by-matthew-davies

Davies, M. (2014). An Agreement to Disagree: The ASEAN Human Rights Declaration and the Absence of Regional Identity in Southeast Asia, Journal of Current Southeast Asian Affairs, Vol.33, No.3, pp. 107-129. Recuperado de: http://nbnresolving.org/urn/resolver.pl?urn:nbn:de:gbv:18-4-8086

Emmers, R. (2016). Enduring Mistrust and Conflict Management in Southeast Asia: An Assessment of ASEAN as a Security Community. Institute for East Asian Studies, Sogang University. Recuperado de: https://www.rsis.edu.sg/staff-publication/enduring-mistrust-andconflict-management-in-southeast-asia-an-assessment-of-asean-as-asecurity- community/\#.XkmY-GhKiM8

Fajardo VAllejo, L.M; TANG, P.S. Y Wilhelmy VON WolfF, M. (2007). El Sudeste asiático: estructura y cambio de sus relaciones 
Intervenir o no intervenir: el desafío de crear un sistema regional de derechos humanos en la ASEAN

ALEJANDRO LAMARQUE

internacionales, Estudios Internacionales, 157, pp. 9-26. Instituto de Estudios Internacionales, Universidad de Chile.

JOYCEE, A.T. Y AMADOR, J.S. III (2016). The role of the Association of Southeast Asian Nations in Post-Conflict Reconstruction and Democracy Support. International Institute for Democracy and Electoral Assistance. Recuperado de: https://www.idea.int/es/publications/catalogue/role-associationsoutheast-asian-nations-post- conflict-reconstruction-and

MERICAN, D. (2007). Cambodia "es Engagement with ASEAN: Lessons for Timor Leste, CICP Working Paper, No.14, pp. 1-23. Cambodian Institute for Cooperation and Peace. Recuperado de: http://www.cicp.org.kh/userfiles/file/Working\%20Paper/CICP\%20w orking $\% 20$ paper $\% 2014-$

$\% 20$ Cambodia's $\% 20$ Engagement $\% 20$ in $\% 20$ ASEAN $\% 20$ by $\% 20$ Din $\% 2$ 0Merican.pdf

Rubiolo, M.F. (2006). Asociación de Naciones del Sudeste Asiático: Construcción de una identidad común. Centro Argentino de Estudios Internacionales. Recuperado de: https:/ / racimo.usal.edu.ar/5838/1/P\%C3\%A1ginas $\% 20$ desde'Tesis. $\mathrm{UNI} \% 270045452$.Asociaci $\%$ C3 $\%$ B3n $\% 20 \mathrm{de} \% 20$ naciones $\% 20 \mathrm{del} \% 20$ sudeste $\% 20$ asi $\%$ C3\%A1 tico.pdf

United Nations Security Council, (2011) „Security Council Press Statement on Cambodia- Thailand Border Situation ${ }^{e}$, Press Release SC/10174, 14 February 2011.

Urgell, J. (2007). Guerra y Paz en el Sudeste Asiático, Papeles de cuestiones internacionales, $\quad N^{\circ} 97, \quad$ pp. 125-138. Recuperado de: https://escolapau.uab.cat/img/programas/alerta/articulos/07articulo 056.pdf 
Comité de ASEAN en Buenos Aires

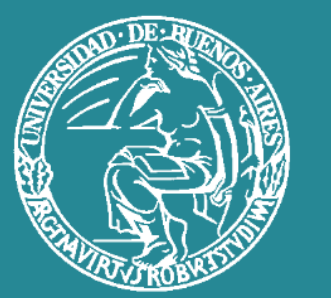

Grupo de Estudios sobre Asia y América Latina Instituto de Estudios de América Latina y el Caribe Universidad de Buenos Aires 